\title{
STUDY OF SYSTEM INTERFACES THROUGH THE NOTION OF COMPLEMENTARITY
}

\author{
Brovar, Yana; \\ Menshenin, Yaroslav; \\ Fortin, Clement \\ Skolkovo Institute of Science and Technology (Skoltech)
}

\begin{abstract}
Understanding emergence is an important goal of system thinking, as it can express both desirable and negative properties of products and systems. Emergence has also a special importance as it has a direct link to the performance of products and systems, and thus has a direct relationship with the quality of life and thus sustainability in our societies. Emergence and system thinking are closely related to engineering design methodologies. In our paper, we develop a more precise definition of emergence through the core principles of systems complementarity that are similarity, irreducibility and sophisticated relationships expressed through the interfaces between systems, subsystems or product components.

We demonstrate the utility of the approach based on an aircraft pylon case study by presenting a detailed definition of an interface design matrix and analyse how pylon subsystems influence emergence. The results have shown that the product can be perfectly represented by a model-based approach supporting interface management and the assessment of system complementarity. In turn, this approach allows to go beyond a qualitative definition of emergence, as it proposes a quantitative approach through the assessment of complementarity.
\end{abstract}

Keywords: Emergence, Complementarity, Systems Engineering (SE), Design for interfaces, Conceptual design

\section{Contact:}

Brovar, Yana

Skolkovo Institute of Science and Technology (Skoltech)

Systems Engineering

Russian Federation

yana.brovar@skoltech.ru 


\section{INTRODUCTION}

Today's interconnected and globalized world heavily relies on systems principles and particularly "emergence", which refers to "what appears, materializes, or surfaces when a system operates" (Crawley et al., 2015). Understanding emergence is the goal and the art of system thinking, as emergence can lead both to desirable properties (for example, a new product with a greater functionality) and to the negative ones (for instance, a natural disaster). Hosseinie and Mahzoon (2011) denote that emergence is a crucial notion in contemporary science. Emergence has a special importance as it has a direct impact on the performance of products and systems and thus has a direct relationship with the quality of life in our societies.

Emergence and system thinking are closely related to engineering design methodologies. There are generally two main approaches to engineering design methodologies, one is based on functions and the other is based on affordances. Function-based theories move from function to form, prescribing the functions that the system components must implement. Conversely, affordance-based methods instruct the designers to analyse the affordances of each component of the system and attempt to avoid negative affordances during the design process. However, the general disadvantage of affordancebased methods is that it is challenging to provide this type of analysis without the determined form. Maier and Fadel outlined the main difference between functions and affordances as: "functions are form independent whereas affordances are form dependent" (2003). Affordance-based method is a step forward to analyse system interaction of specific design solutions with a functional environment. In turn, the study of emergent behavior of systems designed by function-based methods could lead to deep analysis of system interaction with a functional environment as well as with its subcomponents.

The notion of emergence has also been explained as system functions that do not reside in any system component, as they are emergent properties of the entire product and cannot be localised to any product component (Maier, 1998). Such interconnectivity between systems requires meticulously keeping track of the interfaces (sub-section 2.1), as their inaccurate management might lead to system failures.

The ability to predict such emergence can result in a competitive advantage, as Mittal and Rainey (2015) defined as the positive occurrence of emergence that fulfils the tasks of a product and maintains the systems components operability in their optimal performance ranges; while negative occurrences that do not meet the goals of the product and exhibit undesirable behaviours, are eliminated. It is also important to be able to quantify and to predict emergence, as it influences the product synthesis defining "how the product will be materialized" (Andreasen et. al., 2015). While emergence is generally represented qualitatively, there is a research opportunity to develop a quantitative approach for evaluating emergence.

In our work, we test the hypothesis that this can be done through the notion of complementarity (subsection 2.2) based on interfaces analysis using the Design Structure Matrix approach (Eppinger and Browning 2012; Browning 2001), studied from a functional perspective as emergence appears in the functional domain. This is realised through the study of engineering to manufacturing system integration, and also the analysis of an aircraft pylon system, where its relationships are studied in detail.

The first form of utility of the approach is that it provides the support of product components representation with an emphasis on the interfaces. The second form of utility is that this design information is expressed by the DSM-based representation providing an analysis of the subsystem impact on emergent properties of the product/system as a whole.

This paper has the following structure. In Section 2 we introduce the concepts of interfaces, relationships and complementarity and explain the methodology that is used throughout the paper. Section 3 is dedicated to the overview of an aircraft pylon case study - and its conceptual modelling representation by means of OPCloud. The design interface matrix and its analysis are presented in Section 4. We discuss the results in Section 5 and present conclusions in Section 6.

\section{INTERFACES, RELATIONSHIPS AND COMPLEMENTARITY}

\subsection{Interfaces and Relationships}

System interfaces contain essential information about the system and its decomposed parts (internal interfaces) as well as between the system and another system (external interfaces). Zeigler (2009) 
highlighted that: "the essence of modelling lies in establishing relations between pairs of system description". By definition, interacting components are connected by some functional and physical interfaces in nature. Interfaces may involve geometric connections between two components (structural and spatial), or may involve flow interactions (described by the transfer of energy, information and material).

Interface management problems arise early in the product development process with the goal of achieving interoperability and physical compatibility between all interconnected system elements. Fosse (2013) emphasised that describing and specifying interfaces in a general way is a challenging task; for this reason the interfaces are defined in a variety of ways in the design literature. The NASA System Engineering Handbook (2016) defines interfaces as "any boundary between one area and another". There are other points of view on the definition of interfaces, such as a logical or physical relationship (Rahmani and Thomson, 2012) or a linkage (Mikkola, 2001).

Significant attempts were made to develop a proper interface representation to help design team members from different disciplines to collaborate. One of these approaches is an integrated multidisciplinary interface model (Zheng et al., 2015), enabling multidisciplinary integration and hierarchical decomposition of mechatronic systems. The main focus of this approach is related to avoiding model inconsistency and proposing common and unified terminology. Our approach aims to allow an analysis of the quantitative dependence of emergence based on the types and number of interfaces presented in this study.

\subsection{Complementarity}

According to Oxford Languages, complementarity can be defined as "a relationship or situation in which two or more different things improve or emphasise each other's qualities". Based on this definition, a few important aspects can be further defined: (1) complementarity is associated with the interfaces (relationship) between the product's subsystem (two or more different things); (2) complementarity is connected with emergence (through the "improvement or emphasising each other's qualities").

The importance of complementarity lies in the ability to connect emergence, which is of a qualitative nature, with interface management, which is a method for quantitative assessment (Browning, 2001). Thus, the notion of complementarity could support the study of system interfaces and ultimately - to bring the quantitative evaluation of emergence.

This notion of complementarity was further developed with the formulation of complementary data structures for Manufacturing Process Management in the research work presented by Gagné and Fortin (2007), Huet et al. (2010) and Fodeh et al. (2012). They demonstrated the complementary approach based on the similar nature of the Engineering Bill of Materials (EBOM) and of the Manufacturing Bill of Materials (MBOM). The EBOM represents the structural decomposition of the product and essentially carries the spatial dimension during the detail design stage. In turn, the MBOM includes all EBOM components, but is structured to carry the manufacturing sequence and therefore is much more time-related. Thus, on the one hand, the MBOM is similar to the EBOM, but in fact it is irreducible as it contains different and supplemental information when compared to the EBOM.

In a similar way, from a system perspective, we thus propose to describe the complementary of two products/systems based on the principles of similarity and irreducibility, combined with the definition of extended or sophisticated relationships between systems entities. The principle of similarity is applied in various fields of science, ranging from biological models (Henkel, 2018) to semantic similarity (Elavarasi et al., 2014), as well as in the field of design research (Menshenin and Crawley, 2018; Alsalehi, 2019). We define similarity at the functional level, thus two systems are considered similar if the purposes of their use are in the same functional domain and thus allow them to be interfaced and put in close relation. Similarity is thus a principle that allows two systems to support and emphasise each other, through their common relationships.

From a system perspective emergence has its roots also in the mutual irreducibility of the models (Hosseinie and Mahzoon, 2011), therefore irreducibility is a proposed second principle of complementarity. Stephan (2002) also highlighted that a system's property is taken to be emergent if it is irreducible. The notion of irreducibility is also widely used in multiple fields of science. For instance, from the philosophy perspective, irreducibility is the principle that a complete account of an entity is not possible at lower levels of explanation. Also, this notion could refer to biology, helping to conclude that some biological systems are too complex and therefore have irreducible complexity to evolve from simpler ones in the process of evolution (Behe, 2006). Thereby, the irreducibility of systems can be seen 
as the impossibility of reduction of one system into the other without losing its essence. For example, in the aerospace domain, a combustion chamber in a jet engine is actually represented by a single part number in the engineering definition (that is in the EBOM), but in manufacturing planning where the MBOM is defined, it is represented by many parts that need to be welded together using various manufacturing processes. For instance, the combustion chamber must be covered by a ceramic coating which will require a complex robotic manufacturing process for the deposition of multiple coats of the ceramic protection. The MBOM is thus structured to define the manufacturing sequence and should also contain information about the manufacturing processes, tools and other metadata. Thus, it is certainly irreducible to the EBOM, as it cannot be reduced to it without losing its own essence.

\subsection{Methodology}

The methodology that is used in our paper consists of three steps. The first step is to define the system of interest and accompanying system (Crawley et al., 2015), which are in our case an airframe, an engine and an aircraft pylon discussed in sub-section 3.1. The second step presented in sub-section 3.2 is to encode the system of interest and the accompanying systems into the conceptual models through OPCloud. The third step is to transfer the design knowledge from the models to the design interface matrix realised through the DSM-based approach and presented in section 4. Such an interface representation model supports the analysis of how the subsystems can be combined to realise emergence. All of these steps are integrated with each other and describe a coherent approach to analyse the subsystems' influence on emergence.

\section{PYLON CASE STUDY AND ITS CONCEPTUAL MODELING REPRESENATION}

\subsection{Pylon overview}

The pylon case study has been used at Ecole Polytechnique of Montreal in collaboration with the Centre d'Adaptation de la Main-oeuvre en Aérospatiale du Québec (CAMAQ) and 3 major aerospace companies as part of a Master's program, to train systems integrators for the aerospace industry (Fortin et al., 2006). The students participating in this major project are required to extensively modify an existing pylon design due to a major engine change on a passenger aircraft. Figure 1 below shows the results of one of these design studies which are carried out over 7 months by a team of 15 students supervised by a group of various engineering specialists from industry.

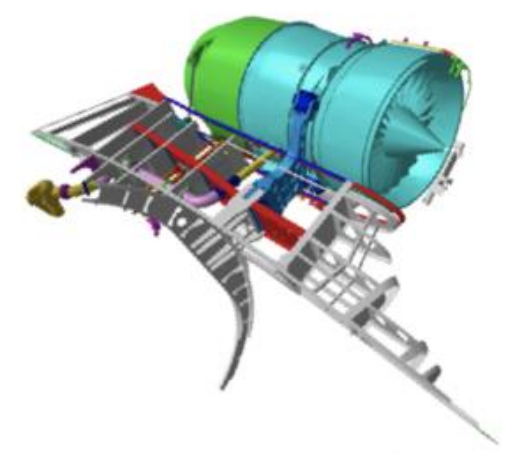

Figure 1. Pylon example

An aircraft pylon is a critical system in any aircraft as it connects the engine to the fuselage (airframe) to meet its primary functions. The engine provides three essential functions: thrust, heating and cooling of the passenger compartment through the bleed air system and also electrical power. The pylon must be designed carefully and must meet a large number of requirements coming from stringent certification rules. The pylon therefore plays the role of a complex interface composed by a set of specific interfaces and relationships between the various components, which express complementarity and insure the positive emergence of the aircraft system.

Since the airframe and engine are in the same aerospace functional domain, it is possible in this case to confirm their similarity by their capability to be put in close relation. The engine is complementary to the airframe by providing its main functions as described above. Also, since the airframe and engine are very 
different by their fundamental nature, it is also easy in this case to confirm their irreducibility. To further assess their complementary, a detailed analysis of their detailed relationships and interfaces is required.

\subsection{Conceptual Modelling of the Pylon System}

There are a few different conceptual modeling languages that can be used during the 0D/1D modeling phase including the Systems Modeling Language (SysML) (Friedenthal et al., 2014) and the ObjectProcess Methodology (OPM) (Dori, 2002), which are both ISO standards. We are exploiting OPM, as this language brings the design paradigm to the earliest stages - those which start with the core entities of the product development. OPM is an ISO standard (ISO 19450, 2015) that captures and represents the knowledge about concept through the simple entities and their relationships.

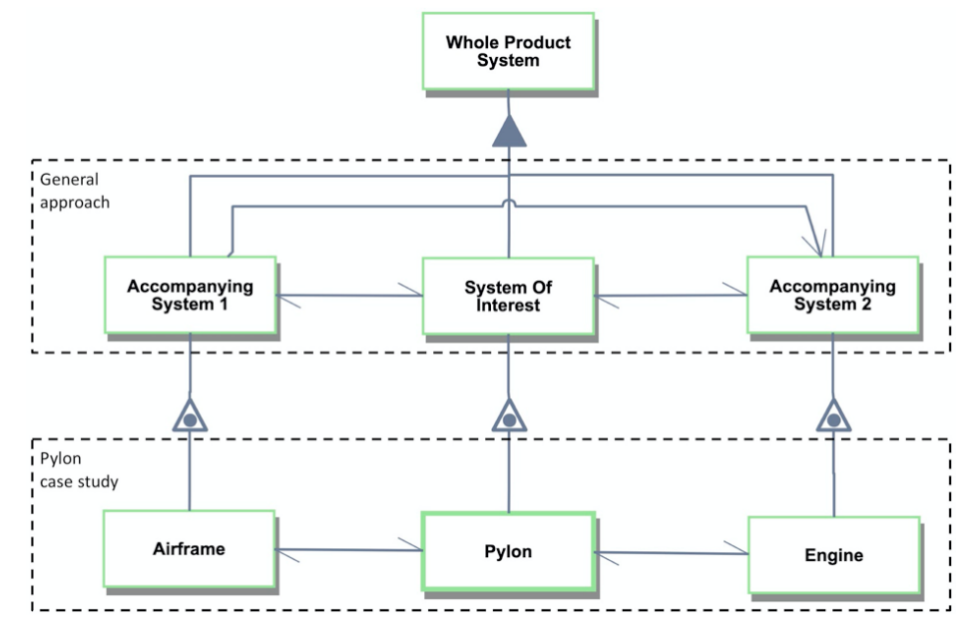

Figure 2. Model-based views: General approach and pylon case study

Figure 2 demonstrates the whole product in OPM notation comprising three systems: the system of interest and two accompanying systems, and also it represents the presence of the interfaces between these systems. The instances of these three entities for the case study are the pylon (system of interest), the airframe (accompanying system 1), and the engine (accompanying system 2). Since Figure 2 is a high-level representation, we only see the external interfaces (the ones which are between the system of interest and accompanying systems). The triangles with black circles inside denote this reality. For example, the airframe is an instance of the accompanying system. And since external relations of the system of interest and accompanying systems are bi-directional, this is a sign that these accompanying systems bring value to each other and, accordingly, complement each other.

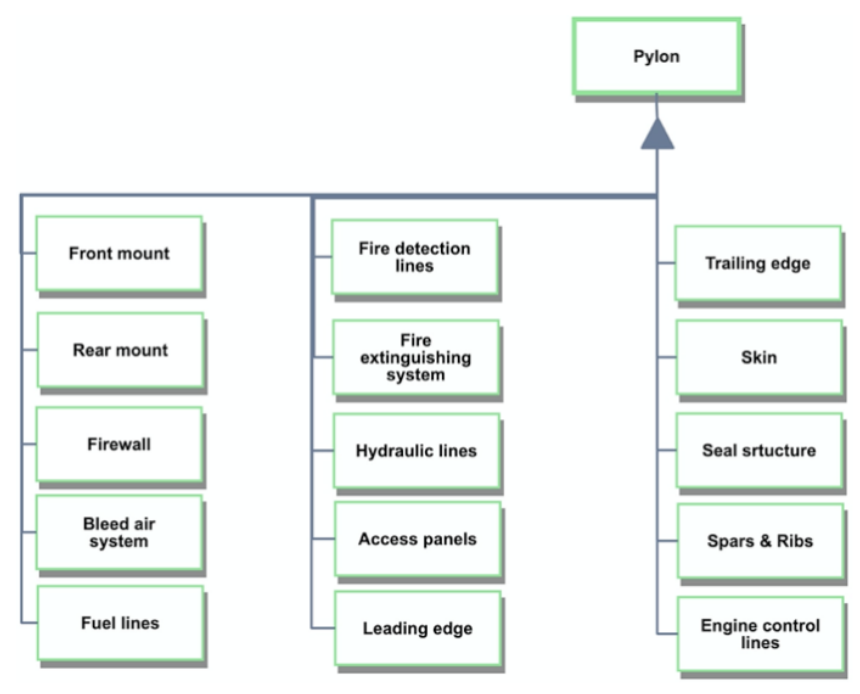

Figure 3. Pylon decomposition

Following the logic presented in Figure 2, the system of interest - the pylon - can be further decomposed into its own components, or 15 subsystems (see Figure 3). For representation through the Object-Process Diagrams (OPDs) the hierarchy and structure of the various models is needed. Thus, 
for greater visualisation and unified representation on one page, we hypothesize that the proposed DSM-based interfaces management model is an appropriate tool to reduce complexity. The utility of this representation is that it can be built for any subsystem keeping the same principles regardless of the level of granularity (Maier et al., 2016).

\section{DESIGN INTERFACE MATRIX}

The design interface matrix (Sosa, 2003) shown in Figure 4 can be described as a special form of DSM representing the interface mapping between the pylon, and the engine and airframe systems. The methodology presented by Pimmler and Eppinger (1994) identifies five types of design interfaces between systems: spatial, structural, energy, material and informational.

The process starts with the interface definition and the decomposition of accompanying systems into subsystems that are relevant to identifying relationships between accompanying systems and the system of interest. Thus, 6 airframe and 6 engine subsystems are defined based on the five types of interfaces. The identically labelled rows and columns of the DSM correspond to the identified subsystems as well as the 15 pylon subsystems.

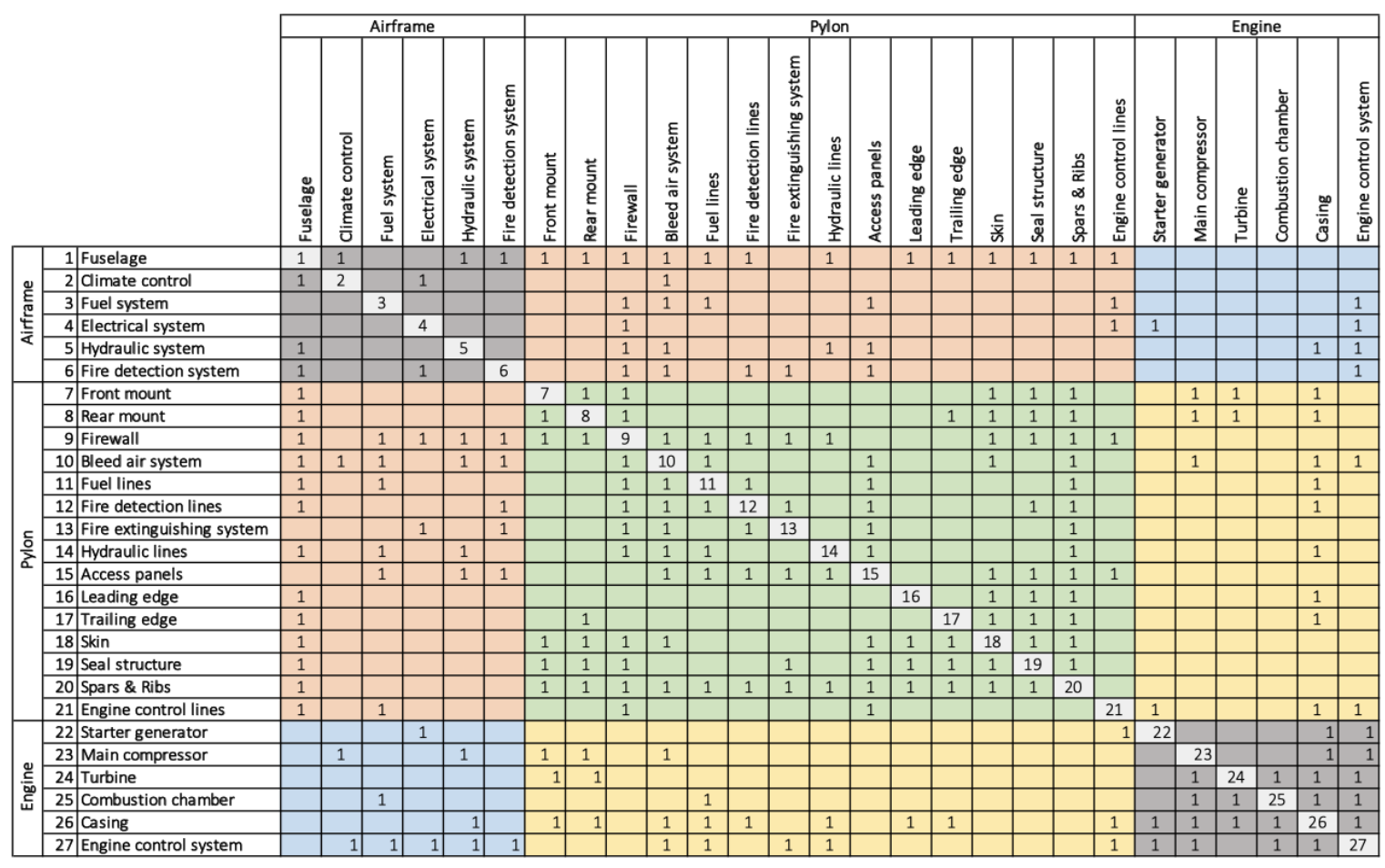

Figure 4. Binary form of design interface matrix for pylon case study

After documenting the minimum required decomposition of the systems, the design interfaces between 15 pylon components and 12 subsystems of accompanying systems (6 airframe and 6 engine subsystems) are identified. Design dependencies are captured by analysing a Preliminary Design Review Report of the aircraft pylon. Figure 4 displays only the binary form of the design interface matrix. That means that the off-diagonal elements of the matrix are marked with 'one' when a given subsystem has at least one type of design interface with another subsystem. Structural and spatial interfaces are bi-directional, however material, information and energy interfaces have the direction of the flow, so they are unidirectional and should be read 'from column to row' in the DSM representation. For example, all firewall subsystem interfaces are spatial or structural, as strong requirements exist on the distance between the firewall and other subsystems without losing accessibility to the required subsystem, so its interface representation is symmetrical about the diagonal. However, the 'electrical system' is essentially unidirectional, as the type of the link describes electrical energy flow, which has a direction from 'electrical system' to the other, such as 'starter-generator on the engine, etc.

To analyse the DSM, we distinguish 9 zones (1 green, 2 grey, 2 yellow, 2 orange, 2 blue). The green zone represents internal interfaces in the system of interest (the pylon), in turn the grey zones represent 
the accompanying engine and airframe systems. They demonstrate the design dependencies of the airframe, the pylon and the engine subsystems, which interconnect the engine and the airframe.

Table 1 shows the number of 'units' in the yellow and orange zones of the design interface matrix, that represents the interfaces of the pylon subsystems to the airframe and the engine (left-hand side), the total sum of those interfaces is equal to 49. In turn, the right-hand side of the table shows the number of interfaces from the airframe and the engine to each of the pylon subsystems. The numbering of subsystems in Table 1 corresponds to the numbering in the design interface matrix (Figure 4).

Table 1. Number of interfaces of each pylon subsystem going to the airframe and the engine (left-hand side) and coming from the airframe and the engine (right-hand side)

\begin{tabular}{|c|l|c|c|c|}
\hline & Subsystem & Pylon to Airframe & Pylon to Engine & Total \\
\hline 7 & Front mount & 1 & 3 & 4 \\
\hline 8 & Rear mount & 1 & 3 & 4 \\
\hline 9 & Firewall & 5 & 0 & 5 \\
\hline 10 & Bleed air system & 5 & 3 & 8 \\
\hline 11 & Fuel lines & 2 & 3 & 5 \\
\hline 12 & Fire detection lines & 2 & 1 & 3 \\
\hline 13 & Fire extinguishing system & 1 & 1 & 2 \\
\hline 14 & Hydraulic lines & 2 & 2 & 4 \\
\hline 15 & Access panels & 3 & 0 & 3 \\
\hline 16 & Leading edge & 1 & 1 & 2 \\
\hline 17 & Trailing edge & 1 & 1 & 2 \\
\hline 18 & Skin & 1 & 0 & 1 \\
\hline 19 & Seal structure & 1 & 0 & 1 \\
\hline 20 & Spars \& Ribs & 1 & 0 & 1 \\
\hline 21 & Engine control lines & 3 & 1 & 4 \\
\hline \multirow{2}{*}{} & Sum & 30 & 19 & 49 \\
\hline
\end{tabular}

\begin{tabular}{|c|c|c|}
\hline Airframe to Pylon & Engine to Pylon & Total \\
\hline 1 & 3 & 4 \\
\hline 1 & 3 & 4 \\
\hline 5 & 0 & 5 \\
\hline 5 & 3 & 8 \\
\hline 2 & 1 & 3 \\
\hline 2 & 1 & 3 \\
\hline 2 & 0 & 2 \\
\hline 3 & 1 & 4 \\
\hline 3 & 0 & 3 \\
\hline 1 & 1 & 2 \\
\hline 1 & 1 & 2 \\
\hline 1 & 0 & 1 \\
\hline 1 & 0 & 1 \\
\hline 1 & 0 & 1 \\
\hline 2 & 1 & 3 \\
\hline 31 & 15 & 46 \\
\hline & & \\
\hline
\end{tabular}

As emergence occurs in the functional domain, we analyse these zones from a functional perspective. The orange zone above the diagonal represents external interfaces from the pylon to the airframe, the sum of which is 30 as presented in Table 1. The orange zone below the diagonal represents 31 external interfaces from the airframe to the pylon. The yellow zone above the diagonal demonstrates external interfaces from the engine to the pylon, the sum of which is 15 (see Table 1). And the yellow zone below the diagonal represents 19 external interfaces from the pylon to the engine. The orange and yellow zones together represent the main engine function of providing thrust, that is in general realised through the interfaces of the Front mount and Rear mount subsystems of the pylon with the Fuselage and the Engine Casing, through which the thrust from the engine is transmitted to the airframe of the aircraft. Thus, the firewall is an important factor in preventing negative emergence in the form of a fire from the engine to the airframe. For this reason, the firewall has five interfaces with airframe subsystems in comparison with the rest of the pylon subsystems, according to Table 1.

The blue zones of the design interface matrix demonstrate external interfaces from the engine to the airframe (zones above the diagonal) and vice versa (zones below the diagonal). The blue zones demonstrate the other two engine functions related to the airframe: climate control, through interfaces of the climate control subsystem, and electrical power generation, through interfaces of the electrical system subsystem.

To quantify emergence, which is a result of the interaction of subsystems, a greater number of relationships would normally lead to higher level of emergence. In Figures 5 we represent the sum interfaces of each pylon subsystem going to the engine and the airframe, that corresponds to the values in the "Total" column in Table 1, and in Figure 6 vice versa (coming from the engine and the airframe to each subsystem of the pylon). From these figures, we may conclude that the bleed air system has the most interfaces with complementary engine and airframe systems, as it has 8 outgoing interfaces and 8 incoming interfaces, so it could influence the system emergency more than other pylon subsystems. 


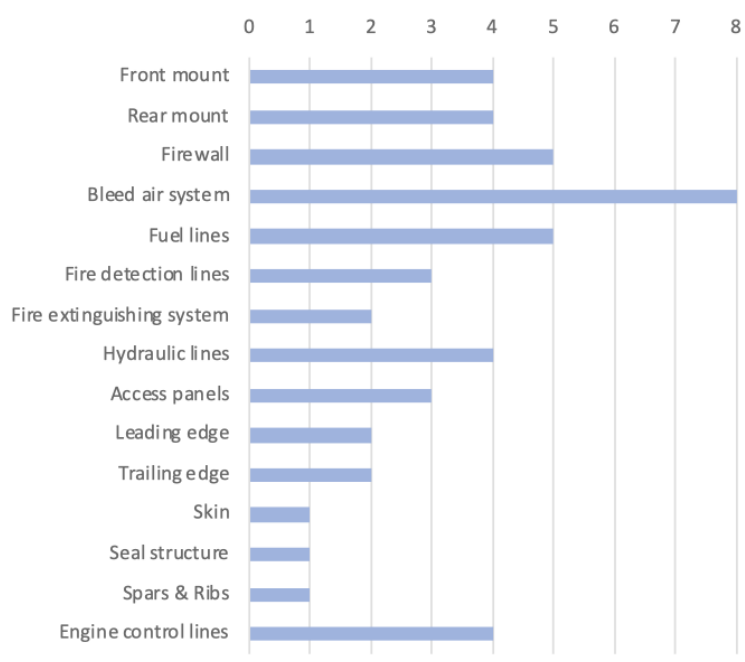

Figure 5. Total number of pylon subsystem interfaces going to the engine and the airframe

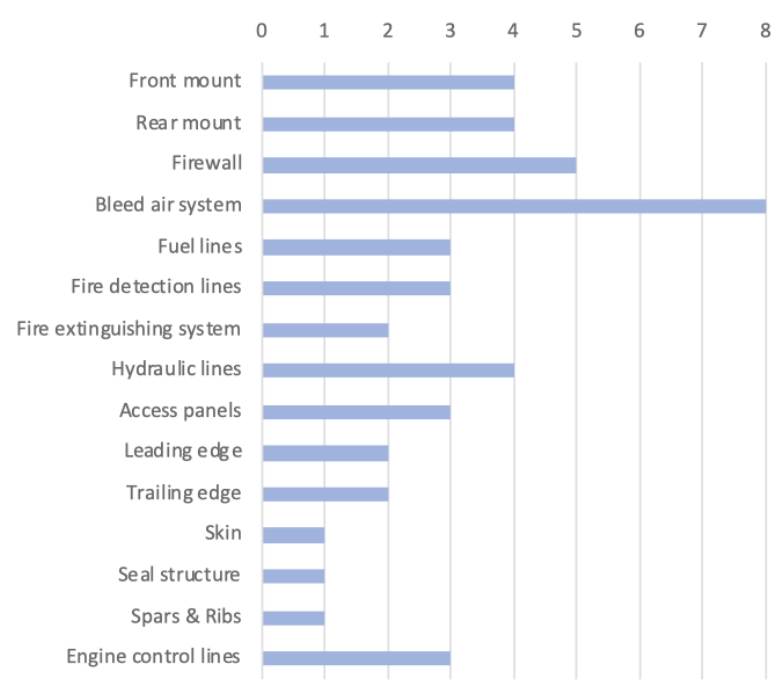

Figure 6. Total number of pylon subsystem interfaces coming from the engine and the airframe

Higher interconnectivity between the bleed air system with the airframe and the engine requires accurate management to prevent system failures. In order to keep track of the interfaces, we apply the bleed air system interfaces representation in OPM notation (Figure 7). This representation consists only of that airframe and engine subsystems, that have interconnection with the bleed air system, so these are not full systems decomposition. The purpose of that representation is to support the analysis of this subsystem impact on emergence, allowing to provide quantitative emergence evaluation. All bleed air system interfaces are bidirectional, meaning that all these pairs of subsystems are complementary.

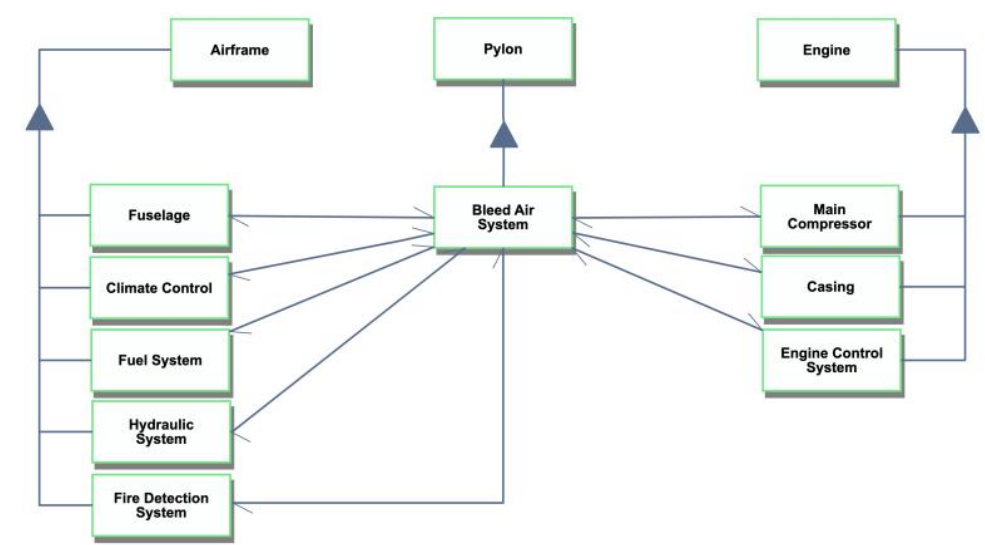

Figure 7. Bleed air system interfaces representation in OPM 


\section{RESULTS AND DISCUSSION}

In this paper, we connected the notion of emergence with complementarity in order to develop a quantitative approach for the evaluation of emergence applicable to complex systems analysis. Ultimately, a better prediction of emergence will lead to designing higher quality products and to decrease the impact of the undesirable results appearing from the product's negative emergence. To achieve these, we define interconnections between complementarity and such related notions as similarity, irreducibility, and relationships through their interfaces between sub-systems.

To reach an appropriate interconnectivity between systems, fostering high quality and avoiding 'negative' emergence, it is required to meticulously keep track of the systems interfaces. To reach this goal, we represented the design knowledge of the aircraft pylon relationships by creating a binary form of the design interface matrix (Figure 4). DSM-based representation provides better visualisation and unified representation of interfaces on one page without using hierarchical structure of design models, helping to reduce complexity. By analysing this DSM through an additional table (Table 1) and diagrams (Figures 5 and 6), we were able to highlight a pylon subsystem which influences the system emergency more than the others. It turned out that the Bleed air system, the main function of which is to cool and heat the passenger compartment, has the largest number of interfaces with the accompanying systems. In order to precisely keep track of the Bleed Air System interfaces preventing system failures we represent its interfaces in OPM notation (Figure 7). According to Figure 7 it can be noted that all interfaces are bi-directional, which means that all pairs of subsystems complement each other. We have also demonstrated that a firewall is an important system feature to prevent the potential 'negative' emergence coming from an engine fire through the pylon.

\section{CONCLUSION}

This research work aims towards the quantification of emergence through the definition of complementarity to realise better functionalities of products and systems, to predict undesirable outcomes and promote quality. Thus, by supporting the notion of complementarity through similarity, irreducibility, and relationships, the evaluation of emergence goes beyond its qualitative nature.

The current limitation is that we do not differentiate the various types of subsystems interfaces (structural, spatial, material, energy and informational) of the product, and their specific impact on emergence. Thus, the direction for future work is to apply the proposed approach with the inclusion of the various types of interfaces. The relationship between emergence and affordances will be also considered as a potential avenue of the study. We are also planning to apply the proposed approach further with similarity and irreducibility quantification, as well as to study other three attributes of the 4Cs model proposed by Pickton and Broderick (2006), such as consistency, coherence and continuity, proposed for integrated marketing communications.

\section{ACKNOWLEDGMENTS}

The authors acknowledge the contributions of Polytechnique Montreal, CAMAQ, the industrial partners and the various student teams that have contributed to this project. The authors also wish to thank Mr. Jean-Marc Rufiange for his contributions through various verbal exchanges to the concept of complementary and specifically irreducibility.

\section{REFERENCES}

Alsalehi, S. (2019), Similarity in Design: A Framework for Evaluation, Master's Thesis, Skolkovo Institute of Science and Technology, Russia. Available at: http://systemarchitect.mit.edu/docs/alsalehi19.pdf

Andreasen, M.M., Hansen, C.T. and Cash, P. (2015), Conceptual design: Interpretations, Mindset and Models, Springer, London.

Behe, J. Michael (2006), Darwin`s Black Box: The Biochemical Challenge to Evolution, New York/London/Toronto/Sydney: Free Press.

Browning, T.R. (2001), “Applying the Design Structure Matrix to System Decomposition and Integration Problems: a Review and New Directions”, IEEE Transactions on Engineering Management, 48, 292-306.

Crawley, E., Cameron, B. and Selva, D. (2015), System architecture: strategy and product development for complex systems, Prentice Hall Press.

Dori, D. (2002), Object-Process Methodology: A Holistic System Paradigm, Springer. 
Elavarasi, S Anitha, A. J., and Menaga, K. (2014), A survey on semantic similarity measure. International Journal of Research in Advent Technology 2(4), pp. 389-398.

Eppinger S.D. and Browning T.R. (2012), Design structure matrix methods and applications, MIT press.

Fodeh, S.J., Haddad, A., Brandt, C., Schultz, M. and Krauthammer, M. (2012), "Enhancing Clustering by Exploiting Complementary Data Modalities in the Medical Domain", IFIP International Conference on Artificial Intelligence Applications and Innovations, Springer, Berlin, Heidelberg, pp. 357-367.

Fortin C., Sanschagrin B., Huet G. and Gagné S. (2006), "The CAMAQ Project: A Design-Build Experience based on a Virtual Immersion in Aerospace Industry practices", Journal World Transactions on Engineering and Technology Education, Volume 5, Issue 2.

Fosse, E. and Delp, C.L. (2013), "Systems engineering interfaces: A model based approach", IEEE Aerospace Conference, 1-8. https://dx.doi.org/10.1109/AERO.2013.6497322

Friedenthal, S., Moore, A. and Steiner, R. (2014), A practical guide to SysML: the systems modeling language, Morgan Kaufmann.

Gagné, S. and Fortin, C. (2007), "Application of the CMII model to an integrated engineering and manufacturing development environment”, International Journal on Interactive Design and Manufacturing (IJIDeM), 1(1), pp. 5-13.

Henkel R., Hoehndorf R., Kacprowski T., Knu C., Liebermeister W., and Waltemath D. (2018), Notions of similarity for systems biology models, vol. 19, no. May 2016, pp. 77-88.

Hosseinie, R. and Mahzoon, M. (2011), "Irreducibility and emergence in complex systems and the quest for alternative insights", Complexity, 17(2), pp. 10-18.

Huet G., Pellerin R., Fortin C., McSorley G., and Toche B. (2010), "Information Structures and Processes to Support Data Exchange between Product Development and Production Planning \& Execution Systems", Journal of Operations and Logistics, 4 (3), pp. 29-38

ISO 19450 (2015), Automation systems and integration — Object-Process Methodology. [online] International Organization for Standardization. Available at: https://www.iso.org/standard/62274.html (accessed date: November 18, 2020).

Maier, J.F., Eckert, C.M. and Clarkson, P.J. (2016), "Model granularity and related concepts", Proceedings of the 14th International DESIGN Conference, Dubrovnik, Croatia.

Maier, M.W. (1998), “Architecting principles for systems-of-systems”, Systems Engineering, 1, pp. $267-284$.

Maier, J.R.A. and Fadel, G.M. (2009), "Affordance-based design methods for innovative design, redesign and reverse engineering”, Research in Engineering Design, 20(4), p. 225.

Menshenin Y. and Crawley E. (2018), "DSM-Based Methods to Represent Specialization Relationships in a Concept Framework", In 20th International DSM Conference, pp. 151-157.

Mikkola, J.H. (2001), "Modularity and interface management of product architectures", International Conference on Management of Engineering and Technology, PICMET'01. Portland, pp. 599-609.

Mittal, S. and Rainey, L. (2015), "Harnessing emergence: the control and design and emergent behavior in system of systems engineering”, SummerSim: summer simulation multi-conference 2015, Chicago, USA.

NASA (2016), Systems Engineering Handbook.

Pickton, D. and Broderick, A. (2006), Integrated Marketing Communications, 3rd edition, Financial Times Prentice Hall

Pimmler, T. U. and Eppinger, S. D. (1994), “Integration analysis of product decompositions”, Working papers 3690-94., Massachusetts Institute of Technology (MIT), Sloan School of Management.

Rahmani, K. and Thomson, V. (2012), “Ontology based interface design and control methodology for collaborative product development", Computer-Aided Design, 44(5), pp. 432-444. https://doi.org/10.1016/j.cad.2011.12.002

Sosa M. E., Eppinger S. D. and Rowles C. M. (2003), "Identifying Modular and Integrative Systems and Their Impact on Design Team Interactions", ASME Journal of Mechanical Design, 125(2), pp. 240-252.

Stephan, A. (2002), "Emergentism, Irreducibility, and Downward causation”, Grazer Philosophishe Studien, vol.65, p. 77-93.

Zeigler B., Muzy A. and Yilmaz L. (2009), “Artificial Intelligence in Modeling and Simulation”, In Meyers R. (eds) Encyclopedia of Complexity and Systems Science. Springer, New York, NY. https://doi.org/10.1007/978-0-387-30440-3_24

Zheng C., Le Duigou J., Bricogne M. and Eynard B. (2015), "Multidisciplinary interface model for design of mechatronic systems", Computers in Industry, 76, pp. 24-37 